\title{
Effect of lactoferrin- and transferrin-conjugated polymersomes in brain targeting: in vitro and in vivo evaluations
}

\author{
Hui-le GAO, Zhi-qing PANG, Li FAN, Kai-li HU, Bing-xian WU, Xin-guo JIANG* \\ Department of Pharmaceutics, School of Pharmacy, Fudan University, Shanghai 201203, China
}

\begin{abstract}
Aim: To evaluate the effect of lactoferrin (Lf) and transferrin (Tf) in brain targeting.
Methods: Polymersomes (PSs), employed as vectors, were conjugated with Lf or Tf and were characterized by morphology, particle size, zeta potential, and surface densities of the Lf or Tf molecules. In vitro uptake of Lf-PS and Tf-PS by bEnd.3 cells was investigated using coumarin- 6 as a fluorescent probe. In vivo tissue distribution and pharmacokinetics of ${ }^{125}$ I-Lf-PS and ${ }^{125}$ I-Tf-PS were also examined. Results: The mean particle size of PS, Lf-PS, and Tf-PS was around $150 \mathrm{~nm}$ and the zeta potential of the PSs was about -20 mV. Less than $0.12 \%$ of the coumarin was released from coumarin-6-loaded PS in $84 \mathrm{~h}$ indicating that coumarin-6 was an accurate probe for the PSs' behavior in vitro. It was shown that the uptake of Lf-PS and Tf-PS by bEnd.3 cells was time-, temperature-, and concentrationdependent. Both Lf and Tf could increase the cell uptake of PSs at $37^{\circ} \mathrm{C}$, but the uptake of Tf-PS was significantly greater than that of Lf-PS. In vivo tissue distribution and pharmacokinetics in mice revealed higher brain uptake and distribution of Tf-PS than Lf-PS, which was in accordance with in vitro uptake results. The drug targeting index (DTI) of Tf-PS with regard to Lf-PS was 1.51.
\end{abstract}

Conclusion: Using a PS as the delivery vector and bEnd.3 cells as the model of the blood-brain barrier (BBB), Tf was more effective than Lf in brain targeting.

Keywords: blood-brain barrier; lactoferrin; transferrin; brain drug targeting

Acta Pharmacologica Sinica (2010) 31: 237-243; doi: 10.1038/aps.2009.199

\section{Introduction}

The blood-brain barrier (BBB), the most formidable obstacle in the treatment of brain diseases, protects the central nervous system (CNS) from exogenous toxicants, but at the same time, also excludes potential therapeutics ${ }^{[1]}$. In fact, almost all of the large-molecule drugs and more than $98 \%$ of small-molecule drugs cannot cross the $\mathrm{BBB}^{[2]}$. Realizing that so few drugs cross the $\mathrm{BBB}$, researchers have continuously committed to developing various drug delivery and targeting strategies to overcome this obstacle ${ }^{[3-5]}$. However, the efforts are still far from sufficient.

As local invasive (direct injection/infusion) delivery has been associated with many disadvantages ${ }^{[6]}$, global noninvasive strategies taking advantage of endogenous nutrient transport systems present at the BBB can facilitate widespread transport across the whole brain without disruption of the barrier properties ${ }^{[7]}$. Among the various noninvasive approaches, receptor-mediated systems seem to be one of the most promis-

\footnotetext{
* To whom correspondence should be addressed.

E-mail xgiang@shmu.edu.cn

Received 2009-08-06 Accepted 2009-12-21
}

ing. Coupling vectors with specific receptors on the BBB to loading vehicles combines the advantages of brain targeting, high incorporation capacity, reduction of side effects, and circumvention of the multidrug efflux system ${ }^{[8]}$.

Lactoferrin (Lf) and transferrin (Tf) belong to the transferrin family. Tf is a single-chain glycoprotein containing about 700 amino acids whereas Lf contains about 690 amino acids ${ }^{[9,10]}$. Though Tf and Lf are quite similar overall in sequence and structure, and coordinate iron in the same manner, they differ in the structure of their inter-lobe linker, the salt bridge between the helical linker, their pattern of disulfide bonding, and their receptor binding properties ${ }^{[11]}$. Lf receptor (LfR) and Tf receptor (TfR) have been demonstrated to exist on the BBB in different species and to be involved in Lf and Tf transport across the BBB in vitro and in vivo ${ }^{[12-15]}$. There are interesting reports that the expression of LfR in the brain is increased under some disease conditions such as Parkinson's disease and Alzheimer's disease ${ }^{[16-18]}$ and that TfR is more highly expressed on tumor cells than on ordinary cells ${ }^{[19]}$. These data suggest Lf and Tf have prospective benefits in brain targeting.

Recently, Ji et al compared the brain uptake of Lf with that of Tf; the uptake of Lf was much higher than Tf, indicating that 
Lf might be more useful as a ligand for facilitating drug delivery into the brain ${ }^{[20]}$. However, there are several factors that can affect the brain targeting of drug delivery systems, such as the binding affinity and capacity of targeting ligands, the surface density of the ligands conjugated with each vector, the characteristics of vectors, and blood elimination. The characteristics of targeting ligands might change after they conjugate with vectors, and the targeting effect of drug delivery systems is not always coincident with that of targeting ligands. Therefore, it has not been confirmed which ligand - Lf or Tf - has better brain targeting ability after conjugation with vectors, which is important for researchers to develop new effective brain drug delivery systems.

To evaluate the effect of two targeting ligands, Tf and Lf, in enhancing drug delivery into the brain, a vector could be employed. Polymersomes (PSs), as a new class of synthetic thin-shelled capsules based on block copolymer chemistry, are self-assembled vesicles of amphiphilic block copolymers with thicker and tougher membranes than lipids ${ }^{[21,22]}$. Compared with liposomes, PSs contain many advantages such as adjustable amphiphile molecular weight (MW) and ratio, tunable physical and chemical properties, and tunable in vivo behavior $^{[23]}$. Pang et al confirmed that PSs could be employed as vectors to develop a brain targeting drug delivery system ${ }^{[23]}$.

The objective of this paper is to develop two brain delivery systems, Lf-conjugated PS (Lf-PS) and Tf-conjugated PS (Tf-PS), and to compare the relative superiority of Lf and Tf in brain drug targeting.

\section{Materials and Methods}

Materials and animals

Poly (butadiene-b-ethylene oxide) (PBD-PEO, 5000-2300) was purchased from Polymer Source Inc, America. Poly (ethylene glycol-b-lactic acid) (PEG-PLA, 3400:4000) and maleimidePEG-PLA (Mal-PEG-PLA, 3400:4000) was custom-synthesized by Chengdu Organic Chemicals Co, Ltd, China. Holo-transferrin (human), lactoferrin (from bovine colostrum), 2-iminothiolane (Traut's reagent) and coumarin- 6 were purchased from Sigma, America. $\mathrm{Na}^{125} \mathrm{I}$ was purchased from Chengdu Gaotong Isotope Corporation, China. Sepharose CL-4B was purchased from Pharmacia, Sweden. Other agents (analytical pure) were all from Sinopharm Chemical Reagent Co, Ltd, China.

KM mice (18-20 g, ㅇ) were obtained from Shanghai Slac Laboratory Animal Co, Ltd and maintained at $22 \pm 2{ }^{\circ} \mathrm{C}$ on a 12-h light-dark cycle with access to food and water ad libitum. The animals used for the experiment were treated according to the protocols evaluated and approved by the ethical committee of Fudan University.

\section{Preparation and characterization of PS, Lf-PS, and Tf-PS Preparation of PSs}

PSs were prepared using film rehydration as described by Photos et $a l^{[24]}$. Briefly, $2 \mathrm{~mL}$ of copolymer solution in dichloromethane (5 g/L, PBD-PEO:PEG-PLA:MAL-PEG-PLA=7:2:1) was first thoroughly dried onto the walls of a $100-\mathrm{mL}$ glass vial for at least $1 \mathrm{~h}$ on the rotary evaporator (R-200, Buchi, Germany). A volume of $2 \mathrm{~mL}$ of $0.01 \mathrm{~mol} / \mathrm{L}$ sodium phosphate buffer (PBS), $\mathrm{pH} 7.4$, was added to rehydrate the film for 30 $\min$ at $37^{\circ} \mathrm{C}$. The liquid was subjected to sonication $(200 \mathrm{~W})$ for $200 \mathrm{~s}$, followed by passing through a $0.22 \mu \mathrm{m}$ microporous membrane. Coumarin-6-loaded PSs were prepared with the same procedure except that $0.1 \%(w / v)$ of coumarin-6 was added to the dichloromethane solution before film formation and the obtained PSs were subjected to a $1.5 \times 20 \mathrm{~cm}$ sepharose CL-4B column and eluted with $0.01 \mathrm{~mol} / \mathrm{L}$ PBS, pH 7.4, to remove the unentrapped coumarin-6.

\section{Preparation of Lf-PS and Tf-PS}

Lf and Tf were thiolated by a reaction for 60 min with a 30:1 molar excess of 2-iminothiolane in $0.15 \mathrm{~mol} / \mathrm{L}$ sodium borate buffer, pH 8.0, supplemented with $0.1 \mathrm{mmol} / \mathrm{L}$ EDTA, as described previously ${ }^{[25]}$. The product was then applied to a Hitrap ${ }^{\mathrm{TM}}$ Desalting column (Pharmacia, Sweden) and eluted with $0.01 \mathrm{~mol} / \mathrm{L}$ PBS, pH 7.4. The protein fractions were collected and introduced thiol groups were determined spectrophotometrically $(\lambda / \mathrm{nm}=412)$ with Ellman's reagent ${ }^{[26]}$.

Lf-PS and Tf-PS were prepared by incubating the purified thiolated Lf or Tf with the PS at room temperature for $5 \mathrm{~h}$. The products were then subjected to a $1.5 \times 20 \mathrm{~cm}$ sepharose CL-4B column and eluted with $0.01 \mathrm{~mol} / \mathrm{L} \mathrm{PBS}, \mathrm{pH} 7.4$, to remove the unconjugated proteins. The particles concentration was determined by turbidimetry using a UV-2401 spectrophotometer at $350 \mathrm{~nm}$ (Shimadzu, Japan).

${ }^{125} \mathrm{I}$-Lf-PS and ${ }^{125} \mathrm{I}$-Tf-PS were prepared by incubating the Lf or Tf with $\mathrm{Na}^{125} \mathrm{I}$ ( $1 \mathrm{mg} 74 \mathrm{MBq}$ and $1 \mathrm{mg} 92 \mathrm{MBq}$, respectively) at $35^{\circ} \mathrm{C}$ for $5 \mathrm{~min}$. The products were applied to a Hitrap Desalting column and eluted with $0.01 \mathrm{~mol} / \mathrm{L}$ PBS, pH 7.4. The protein fractions were subjected to the above procedure to prepare ${ }^{125}$ I-Lf-PS and ${ }^{125}$ I-Tf-PS.

\section{Morphology, particle size, and zeta potential}

The mean diameter and zeta potential of the PS, Lf-PS, and Tf-PS were determined by dynamic light scattering (DLS) using a Zeta Potential/Particle Sizer NICOMPTM380 ZLS (Santa Barbara, CA). The morphological examination of PS was carried out by transmission electron microscope (H-600, Hitachi, Japan).

\section{The number of Lf or Tf molecules conjugated with each Lf-PS or Tf-PS}

The calculation for the surface number of Lf or Tf molecules per Lf-PS or Tf-PS was based on the relation between concentration of Lf-PS or Tf-PS and intensity of radioactivity of Lf or $\mathrm{Tf}^{[25,27]}$. The procedure is as follows:

If $\mathrm{A}_{1}$ is the intensity of radioactivity of ${ }^{125} \mathrm{I}-\mathrm{Lf}$ or ${ }^{125} \mathrm{I}-\mathrm{Tf}, \mathrm{m}_{1}$ is the weight of Lf or Tf; then the specific radioactivity (k1) can be calculated by: $\mathrm{k}_{1}=\mathrm{A}_{1} / \mathrm{m}_{1}$;

If $\mathrm{m}_{2}$ is the weight of ${ }^{125} \mathrm{I}$-Lf-PS or ${ }^{125} \mathrm{I}$-Tf-PS; $\mathrm{D}$ is the particle diameter of ${ }^{125} \mathrm{I}$-Lf-PS or ${ }^{125} \mathrm{I}$-Tf-PS; then the number $\left(\mathrm{N}_{1}\right)$ of ${ }^{125} \mathrm{I}$ Lf-PS or ${ }^{125}$ I-Tf-PS can be calculated by $\mathrm{N}_{1}=6 \times \mathrm{m}_{2} \times 10^{-3} /\left[\Pi \times\left(\mathrm{D}^{3}-\right.\right.$ $\left.\left.\mathrm{d}^{3}\right) \times 10^{-21} \times \rho\right]$, where $\rho=1.06 \mathrm{~g} / \mathrm{cm}^{3}, \mathrm{~d}=8 \mathrm{~nm}^{[28]}$; 
If $\mathrm{A}_{2}$ is the intensity of radioactivity of ${ }^{125} \mathrm{I}$-Lf-PS or ${ }^{125} \mathrm{I}$-Tf-PS; $\mathrm{M}$ is molecular weight of Lf or Tf; then the number of ${ }^{125} \mathrm{I}-\mathrm{Lf}$ or ${ }^{125}$ I-Tf conjugated with PS can be calculated by: $\mathrm{N}_{2}=\mathrm{A}_{2} \times \mathrm{k}_{1}$ / $\mathrm{M} \times 6.02 \times 10^{23}$;

Then, the number of Lf or Tf per ${ }^{125}$ I-Lf-PS or ${ }^{125}$ I-Tf-PS can be calculated by: $n=N_{2} / N_{1}$.

\section{In vitro release of coumarin-6 from PS}

In vitro release experiments of coumarin-6 from the PS were performed at $37^{\circ} \mathrm{C}$ in $0.01 \mathrm{~mol} / \mathrm{L}$ PBS ( $\mathrm{pH} 4$ and $\mathrm{pH} 7.4$ ) to evaluate if the fluorescent probe remained associated with the particles during an 84-h incubation period. $\mathrm{pH} 4$ and $\mathrm{pH} 7.4$ represented the $\mathrm{pH}$ in the endo-lysosomal compartment and physiologic $\mathrm{pH}$, respectively. Coumarin-6-loaded PSs were incubated at a particle concentration of $7 \mathrm{mg} / \mathrm{L}$ with shaking at $100 \mathrm{r} / \mathrm{min}$ under a predetermined sink condition. Periodic samples were lyophilized, then reconstituted by $1 \mathrm{~mL}$ methanol, and used for HPLC analysis of coumarin- 6 after centrifugalization at $10000 \times \mathrm{g}$ for $10 \mathrm{~min}$.

In vitro uptake of coumarin-6-labeled PS, Lf-PS, and Tf-PS by bEnd. 3 cells

\section{Cell culture}

bEnd. 3 cells, the immortalized mouse brain endothelial cell line, were maintained in 10-cm tissue culture dishes in Dulbecco's Modified Eagle's Medium supplemented with $10 \%$ fetal bovine serum (FBS), penicillin $(100 \mathrm{kU} / \mathrm{L})$ and streptomycin (100 mg/L).

\section{Fluorescent microscopy of PS, Lf-PS, and Tf-PS uptake by bEnd.3} cells

bEnd. 3 cells were seeded at a density of $10^{4}$ cells $/ \mathrm{cm}^{2}$ on a polylysine-coated glass cover slip. On the second day, after preincubation with HBSS for $15 \mathrm{~min}$, the cells were incubated with coumarin-6-loaded PS, Lf-PS, and Tf-PS suspensions (300 mg/L in HBSS, $\mathrm{pH} 7.4$ ) for $0.5,1$, and $2 \mathrm{~h}$ at $37^{\circ} \mathrm{C}$, respectively ${ }^{[29,30]}$. At the end of the experiment, the cells were washed three times with PBS and fixed by $4 \%$ paraformaldehyde for $20 \mathrm{~min}$. Then, they were washed three times with PBS, mounted in Dako fluorescent mounting medium and observed under a fluorescent microscope (Olympus, Japan).

\section{Quantitative analysis of coumarin-6-labeled PS, Lf-PS, and Tf-PS uptake}

bEnd. 3 cells were seeded at a density of $10^{4}$ cells $/ \mathrm{cm}^{2}$ onto 24-well plates. On the second day, after preincubation with HBSS for $15 \mathrm{~min}$, the medium was replaced with the suspension of PS, Lf-PS, or Tf-PS (10-600 mg/L) and incubated for $1 \mathrm{~h}$ at $4{ }^{\circ} \mathrm{C}$ and $1 \mathrm{~h}$ at $37^{\circ} \mathrm{C}$. In a separate experiment, to study the effects of incubation time on particle uptake, the medium was replaced with $1 \mathrm{ml} 100 \mathrm{mg} / \mathrm{L}$ suspension of PS, Lf-PS, or Tf-PS in HBSS per well and the plate was incubated for $15 \mathrm{~min}, 30 \mathrm{~min}, 1 \mathrm{~h}$, and $2 \mathrm{~h}$ at $37^{\circ} \mathrm{C}$. At the end of the incubation period, the cells were washed with ice-cold PBS five times. Subsequently, the cells were solubilized in $400 \mu \mathrm{L} 1 \%$ Triton X-100 and $20 \mu \mathrm{L}$ cell lysate from each well was used to determine the total cell protein content using the BCA protein assay (Shanghai Shenergy Biocolor Bioscience and Technology Co, Ltd, China). A volume of $200 \mu \mathrm{L}$ of the cell lysates were lyophilized and used for HPLC analysis of coumarin-6 after extraction by $700 \mu \mathrm{L}$ methanol. The uptake of PS, Lf-PS, and Tf-PS by bEnd.3 cells was calculated from the standard curve and expressed as the amount of PS, Lf-PS and Tf-PS (mg) taken up per gram of cell protein.

\section{Pharmacokinetics and tissue distribution of ${ }^{125} \mathrm{I}$-Lf-PS and ${ }^{125} \mathrm{I}$-Tf- PS}

A total of 145 mice were randomly divided into two groups, receiving ${ }^{125} \mathrm{I}$-Lf-PS or ${ }^{125} \mathrm{I}$-Tf-PS. The animals were injected in the tail vein at a dose of $20 \mathrm{mg} / \mathrm{kg}{ }^{125} \mathrm{I}$-Lf-PS or ${ }^{125} \mathrm{I}$-Tf-PS. At $0.083,0.25,0.5,1,2,4,6,8,12,16,24$, and $48 \mathrm{~h}$ following iv injection, the blood samples were collected and the mice were sacrificed. The brain, heart, liver, spleen, lung, and kidney tissues were harvested, followed by a quick washing with cold saline and then subjected to weighing and detection by radioimmune $\gamma$ detector (SN-695, Hesuo Rihuan, China).

Concentration data were dose-normalized and plotted as drug concentration-time curves in the blood and brain. The $C_{\max }$ and $t_{\max }$ values were read directly from the concentrationtime profile and the area under the concentration-time curve (AUC) was calculated by the trapezoidal rule. The statistical differences between Lf-PS and Tf-PS were assessed using a paired Student's $t$-test and a $P$ value of less than 0.05 was accepted as significant.

\section{Results}

Preparation and characterization of PS, Lf-PS, and Tf-PS

The mean particle size of PSs was around $150 \mathrm{~nm}$ and the average zeta potential was around $-20 \mathrm{mV}$, which is regarded as favorable for brain transport. After Lf or Tf conjugation, the particle size and zeta potential changed slightly. The mean particle size of Lf-PS and Tf-PS changed from $166.4 \mathrm{~nm}$ and $141.0 \mathrm{~nm}$ to $167.6 \mathrm{~nm}$ and $146.1 \mathrm{~nm}$, respectively, while the average zeta potential of Lf-PS and Tf-PS changed from -24.25 $\mathrm{mV}$ to $-22.08 \mathrm{mV}$ and $-20.45 \mathrm{mV}$, respectively. TEM photographs showed PSs were generally spherical and of regular size (Figure 1).

The average number of Lf or Tf molecules conjugated with

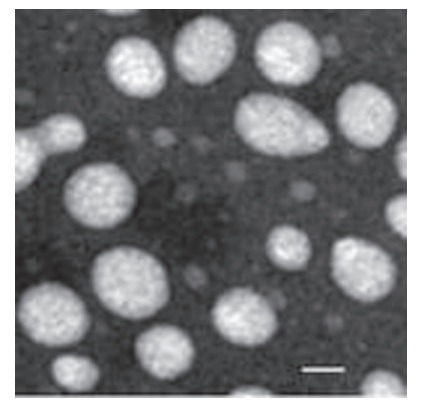

Figure 1. Transmission electron micrograph of PS negatively stained with uranyl acetate solution. The bar is $50 \mathrm{~nm}$. 
PSs was 45.5 and 50.4 per particle, respectively. Therefore, the material ratio was slightly changed in the experiment thereafter to make sure the average molecular number of $\mathrm{Lf}$ and $\mathrm{Tf}$ were as close to each other as possible.

The particle size and zeta potential of particles did not change much after conjugation with Lf or $\mathrm{Tf}$, and the amount of protein conjugated with particles was extremely little compared with the amount of particles and it would not affect the permeability of the membrane of particles. Thus, the in vitro release experiments of coumarin-6 from the PSs can represent that from Lf-PS and Tf-PS. Coumarin-6 has high fluorescence efficiency, liposolubility and detection sensibility, and coumarin-6 has a detection limit as low as $4 \mathrm{ng} / \mathrm{L}$ by HPLC. Many researchers have used it as a fluorescence probe of nano drug delivery systems in quality and quantity analysis ${ }^{[5,27]}$. Although the release speed at $\mathrm{pH} 4.0$ was quicker than that at $\mathrm{pH} 7.4$, the cumulative released percentage of both was smaller than $0.12 \%$ (Figure 2), which is similar to other studies $^{[5,23,27]}$. This suggested that most of the incorporated coumarin- 6 remained in the particles and indicated that the fluorescence signal detected in the cell or tissue samples was attributed mainly to the coumarin- 6 encapsulated into the particles.

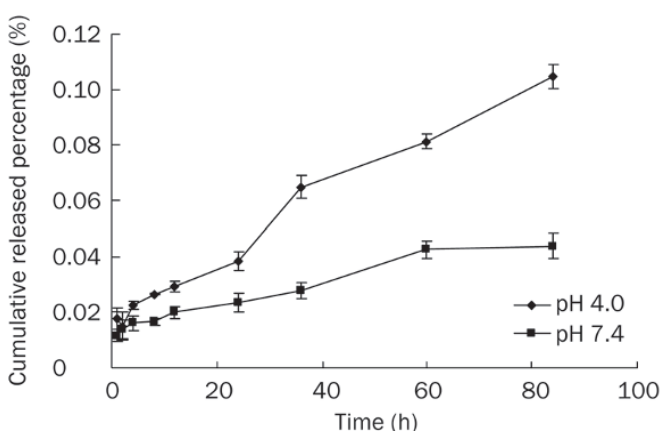

Figure 2. In vitro release of 6-coumarin from PS in $0.01 \mathrm{~mol} / \mathrm{L} \mathrm{PBS}(\mathrm{pH} 7.4$ and 4.0).

Uptake of coumarin-6-loaded PS, Lf-PS, and Tf-PS by bEnd.3 cells The uptake of PS, Lf-PS, and Tf-PS by bEnd. 3 cells was dependent on the incubation time within $2 \mathrm{~h}$ (Figure 3B). At each timepoint, the uptake amount of Lf-PS and Tf-PS was higher than that of PS, about 1.56 and 1.86 times higher than that of $\mathrm{PS}$ at $2 \mathrm{~h}$, respectively; and the uptake amount of Tf-PS was 1.19 times as much as that of Lf-PS. The uptake amount of all PS, Lf-PS and Tf-PS under $37^{\circ} \mathrm{C}$ was much higher than that under $4{ }^{\circ} \mathrm{C}$, suggesting that the uptake of all particles was temperature-dependent (Figure 3A). The uptake of PS, Lf-PS, and Tf-PS was also concentration-dependent. At $37^{\circ} \mathrm{C}$, the uptake increased with an increase in the concentration, showing almost first-order kinetics (Figure 3A). The uptake of Lf-PS and Tf-PS was 2.19- and 3.33-fold greater than that of PS at $600 \mathrm{mg} / \mathrm{L}$, respectively; and the uptake of Tf-PS was 1.52 times greater than that of Lf-PS at $600 \mathrm{mg} / \mathrm{L}$. Under $4{ }^{\circ} \mathrm{C}$, the
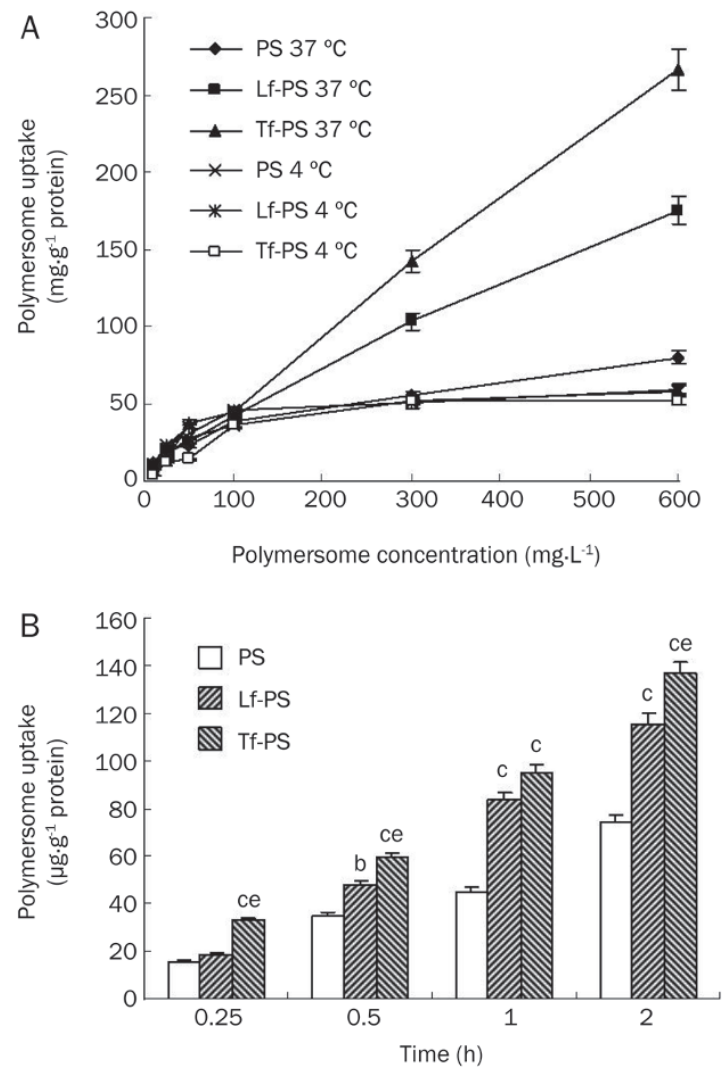

Figure 3. BCECs uptake (A) $10-600 \mathrm{mg} / \mathrm{L} \mathrm{PS}$, Lf-PS, and Tf-PS at $37^{\circ} \mathrm{C}$ and $4{ }^{\circ} \mathrm{C}$ incubation for $1 \mathrm{~h}$, respectively; (B) $100 \mathrm{mg} / \mathrm{L}$ PS, Lf-PS, and Tf-PS at $37{ }^{\circ} \mathrm{C}$ incubation for different time. $n=3$. Mean \pm SD. ${ }^{b} P<0.05$, ${ }^{\mathrm{c}} P<0.01$ vs PS. ${ }^{\mathrm{e}} P<0.05$ vs Lf-PS.

uptake of PS, Lf-PS, and Tf-PS quickly reached saturation at a concentration of $100 \mathrm{mg} / \mathrm{L}$, showing no significant difference between the three kinds of PSs.

Fluorescent microscopy photographs of bEnd.3 cells exposed to PS, Lf-PS, and Tf-PS at the same concentration (300 $\mathrm{mg} / \mathrm{L}$ ) demonstrated that the increase of fluorescent intensity in the cells correlated with an increase in the time of incubation (Figure 4). There was an obvious accumulation of dye of Lf-PS and Tf-PS in the cells compared with that of PS for 30, 60 , and $120 \mathrm{~min}$ at $37^{\circ} \mathrm{C}$. Our in vitro release results confirmed the relative inertia of the coumarin- 6 in the particles. Thus, we concluded coumarin- 6 detected in the cells reflected the particles.

\section{Tissue distribution of ${ }^{125}$-Lf-PS and ${ }^{125}$-Tf-PS}

To evaluate the brain uptake and tissue distribution of the Lf-PS and Tf-PS, ${ }^{125} \mathrm{I}$ was labeled on Lf and Tf, and the blood and other tissues' concentrations of the particles were detected with a radioactive method. Both Lf-PS and Tf-PS exhibited similar concentration-time profiles (Figure 5A). However, the blood $\mathrm{AUC}_{0-\mathrm{t}}$ of Tf-PS was much higher than that of Lf-PS, about 1.84-fold greater (Table 1). The elimination rate constant ( $\beta$ ) of Lf-PS was 3.12-fold greater than that of Tf-PS, and the distribution rate constant $(\mathrm{a})$ of Lf-PS was 2.11-fold greater 



Figure 4. Cell uptake of polymersomes by bEND. 3 cells. $300 \mathrm{mg} / \mathrm{L}$ PS at $37^{\circ} \mathrm{C}$ for $30 \mathrm{~min}(\mathrm{~A}), 60 \mathrm{~min}(\mathrm{D})$, $120 \mathrm{~min}(\mathrm{G}) ; 300 \mathrm{mg} / \mathrm{L}$ Lf-PS for 30 $\min (B), 60 \min (E), 120 \min (H)$ and $300 \mathrm{mg} / \mathrm{L}$ of Tf-PS for $30 \mathrm{~min}(\mathrm{C}), 60$ $\min (\mathrm{F}), 120 \mathrm{~min}(\mathrm{I})$, respectively, bar $50 \mu \mathrm{m}$.
Table 1. Pharmacokinetic parameters of Lf-PS and Tf-PS in whole blood and brain following iv injection of ${ }^{125}$ I-Lf-PS or ${ }^{125}$ I-Tf-PS in mice.

\begin{tabular}{|c|c|c|c|c|}
\hline \multirow{2}{*}{$\begin{array}{l}\text { PK } \\
\text { parameters }\end{array}$} & \multicolumn{2}{|l|}{ Blood } & \multicolumn{2}{|l|}{ Brain } \\
\hline & Lf-PS & Tf-PS & Lf-PS & Tf-PS \\
\hline$\alpha^{a}\left(h^{-1}\right)$ & 0.107 & 0.0508 & - & - \\
\hline$t_{1 / 2 \alpha^{a}}(\mathrm{~h})$ & 6.49 & 13.6 & - & - \\
\hline$\beta^{b}\left(h^{-1}\right)$ & 2.43 & 0.780 & 0.0216 & 0.0223 \\
\hline$t_{1 / 2 \beta}^{\mathrm{b}}(\mathrm{h})$ & 0.285 & 0.888 & 32.1 & 31.1 \\
\hline$t_{\max }(\mathrm{h})$ & 0 & 0 & 0.25 & 0.5 \\
\hline $\begin{array}{c}C_{\max }(\mathrm{mg} / \mathrm{L} \\
\text { or } n g / g)\end{array}$ & $55.68 \pm 5.32$ & $49.86 \pm 5.80$ & $43.44 \pm 6.88$ & $80.04 \pm 7.02^{c}$ \\
\hline $\begin{array}{l}\mathrm{AUC}_{0-\mathrm{t}}(\mathrm{mg} / \mathrm{L} \cdot \mathrm{h} \\
\text { or } \mathrm{ng} / \mathrm{g} \cdot \mathrm{h})\end{array}$ & $219.5 \pm 25.6$ & $403.8 \pm 49.17^{c}$ & $458.6 \pm 89.5$ & $1277 \pm 143^{\circ}$ \\
\hline
\end{tabular}

a: $\alpha$ and $t_{1 / 2 \alpha}$ denotes distribution rate constant and distribution half life respectively; $\mathrm{b}$ : $\beta$ and $t_{1 / 2 \beta}$ denotes elimination rate constant and elimination half life respectively; $C_{\max }$ and $\mathrm{AUC}_{0-\mathrm{t}}$ are Mean $\pm \mathrm{SD} ;{ }^{\circ} P<0.01 \mathrm{vs}$ Lf-PS.

than that of Tf-PS (Table 1).

The brain uptake of Tf-PS was higher than that of Lf-PS at every timepoint (Figure $5 \mathrm{~B}$ ). The $\mathrm{AUC}_{0-\mathrm{t}}$ of brain concentration of Tf-PS was about 2.79-fold greater than that of Lf-PS (Table 1). However, in other tissues, this was not the case. The $\mathrm{AUC}_{0-\mathrm{t}}$ in heart, kidney, and lung of Tf-PS were significantly higher than that of Lf-PS (Figure $5 \mathrm{C}$ ); the $\mathrm{AUC}_{0-\mathrm{t}}$ in spleen tissue had no significant difference with that in brain tissue; but the liver $\mathrm{AUC}_{0-\mathrm{t}}$ of Lf-PS was 1.34 times greater than that of Tf-PS, indicating that the Lf-PS was more easily phagocytized by macrophages than Tf-PS, which could make the blood $\mathrm{AUC}_{0-\mathrm{t}}$ lower.

To determine the difference between Lf-PS and Tf-PS in brain targeting, the drug targeting index (DTI) was calculated:

$\mathrm{DTI}=\left(\mathrm{AUC}_{\text {brain }} / \mathrm{AUC}_{\text {blood }}\right)_{\text {Tf-PS }} /\left(\mathrm{AUC}_{\text {brain }} / \mathrm{AUC}_{\text {blood }}\right)_{\mathrm{Lf}-\mathrm{PS}}$

The closer a compound's DTI is to 1 , the less targeting effect it has ${ }^{[31]}$. The brain DTI of Tf-PS with regard to Lf-PS was 1.51, which indicated that Tf-PS was more effective than Lf-PS in brain targeting.

\section{Discussion and conclusion}

bEnd. 3 cells are an immortalized mouse brain endothelial cell line exhibiting endothelial properties. The cells express von Willebrand factor, vascular endothelial growth factor receptors and can internalize acetylated low-density lipoprotein ${ }^{[32]}$. They are an attractive candidate as a model of the BBB due to their rapid growth, maintenance of blood-brain barrier characteristics over repeated passages, formation of functional barriers and amenability to numerous molecular interventions ${ }^{[5,33]}$. Thus, bEnd. 3 cells were chosen as an easy BBB model to study the brain delivery properties of Lf-PS and Tf-PS in vitro.

The time-, temperature-, and concentration-dependent uptake of the particles suggested a process of active endocytosis. The enhanced uptake of Lf-PS and Tf-PS compared to PS by bEnd. 3 cells might be caused by an additional endocytosis mechanism involving Lf and Tf, and this has been demonstrated by several researchers ${ }^{[5,15]}$. The results also showed that Tf was more effective than Lf in facilitating cell uptake of PSs, which might have several reasons including the following: (1) The dissociation constant of Tf and TfR was approximately $1 \mathrm{nmol} / \mathrm{L}$ but the dissociation constant of Lf with LfR was $7 \mathrm{nmol} / \mathrm{L}$ and $5 \mu \mathrm{mol} / \mathrm{L}$ (LfR has two Lf bind- 

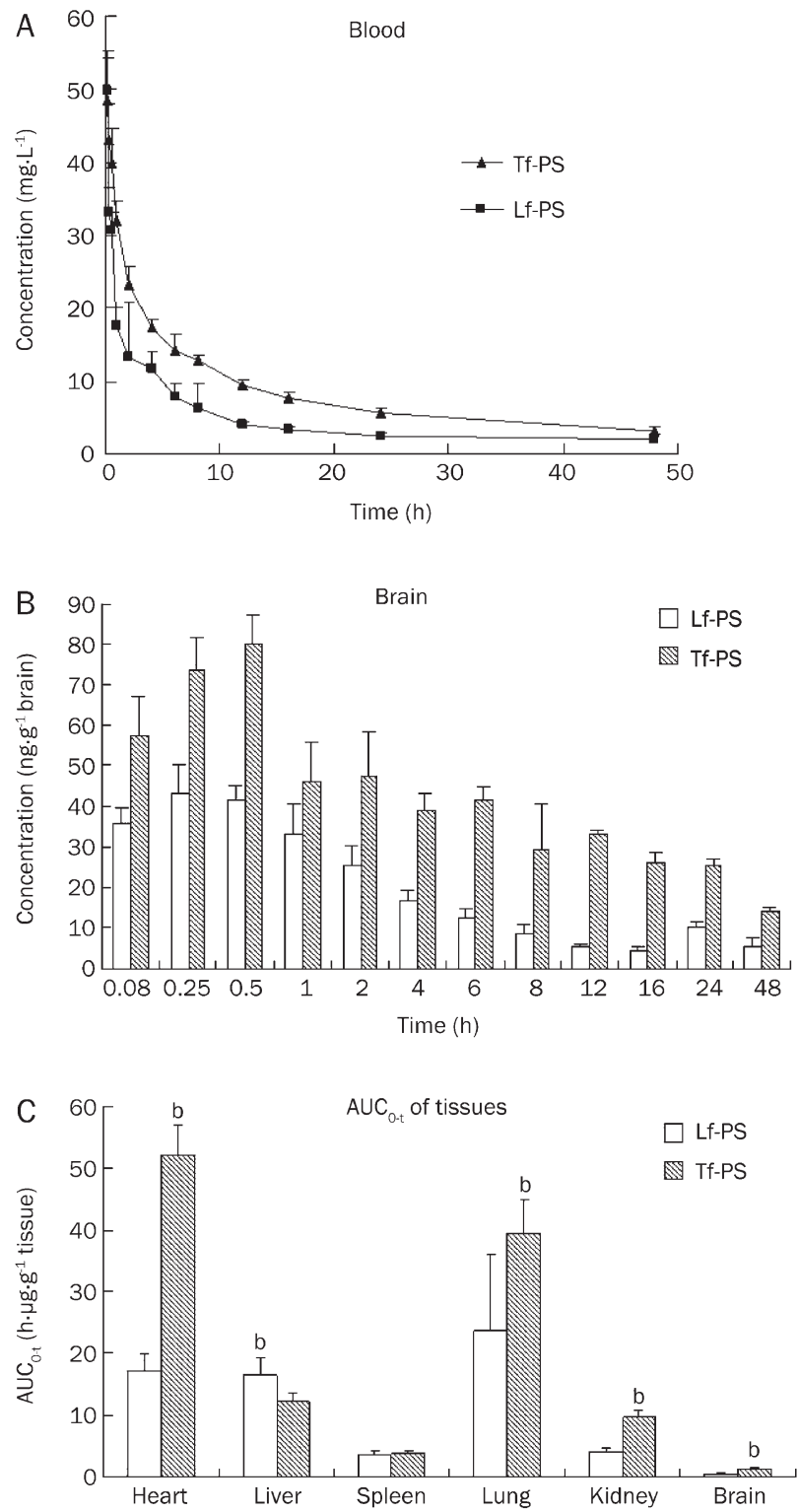

Figure 5. (A) The average PS's concentration-time curve in whole blood followed by iv injection of Lf-PS or Tf-PS in mice. $n=6$. Mean \pm SD. (B) The Lf-PS and Tf-PS concentration in brain at different time; (C) The Lf-PS and Tf-PS AUC ${ }_{0-t}$ in different tissues. ${ }^{b} P<0.05,{ }^{c} P<0.01$ vs Lf-PS.

ing sites ${ }^{[15,34]}$, which means the affinity of $\operatorname{Tf}$ with TfR is much higher than that of Lf with LfR bEnd.3; (2) bEnd.3 cells may exhibit a greater amount of TfR than LfR; and (3) the conjugated PSs down-regulated the affinity of Lf with LfR more than that of Tf with TfR. The last two hypotheses should be identified by further experiments.

TfR and LfR have been demonstrated to exist on the BBB in different species and to be involved in Tf and Lf transport across the BBB in vitro and in vivo ${ }^{[11,12,14,15]}$. In the standard protocol, perfusion is performed before tissues are harvested, and in most of our past experiments, perfusion was performed. In this study, considering the aim was to compare the relative effect of $\mathrm{Tf}$ and Lf, and the blood effect may be neu- tralized, the perfusion was omitted. Several studies published recently have also omitted perfusion ${ }^{[35]}$. Our results showed that the difference of particles' $\mathrm{AUC}_{0-\mathrm{t}}$ of brain was partly affected by the blood elimination, which meant that Lf-PS was more easily identified and eliminated by cells of the mononuclear phagocytic system (MPS). The concentration of circulating Tf is about $25 \mu \mathrm{mol} / \mathrm{L}^{[34]}$, whereas the plasma concentration of endogenous Lf is approximately $16 \mathrm{nmol} / \mathrm{L}^{[20]}$ : $1000-$ fold lower than Tf. The added Lf and Tf (conjugated with PSs) in the blood were about $14 \mathrm{nmol} / \mathrm{L}$ at the peak. Therefore, the added Lf greatly changed the circulating amount of Lf, whereas that of Tf only changed a little, which may have caused the MPS to more easily eliminate the Lf-PS, but the definite mechanism was unclear. Tf-PS was still better than Lf-PS in brain targeting, which was coincident with the results of bEnd. 3 cells uptake in vitro. However, the ligands' conjugation with PSs increased not only the brain uptake but also the liver and spleen uptake of drug delivery systems, which might cause increased toxicities to these organs, a general obstacle that the nanoparticle systems have faced. Considering the BBB prevents almost $100 \%$ of large molecule drugs and about $98 \%$ of small molecule drugs from entering the brain, researchers mainly focused on increasing the brain drug concentration while ignoring other tissues' drug concentrations. Nowadays, researchers have gradually realized that increasing the sitespecific distribution to the brain and decreasing the distribution to other tissues have become the key point in the treatment of brain diseases. Unfortunately, researchers have only been able to increase the distribution of nanoparticle systems to the brain while hardly decreasing the distribution to other tissues, which is the main obstacle researchers are unceasingly devoted to overcoming in brain drug delivery systems.

The brain targeting property of drug delivery systems is largely affected by the targeting ligands, so it is very important to choose the right targeting ligand in research design. Recently, most of the brain targeting moieties are either the related ligands of known receptors, or carriers on the $\mathrm{BBB}$ or the cationic proteins, which can bind with the anionic BBB, such as transferrin receptor monoclonal antibody OX26, insulin, and cationic albumin ${ }^{[23,27,36]}$. However, these targeting ligands generally present problems such as low specificity and not ideal brain targeting properties. Several researchers have tried to select peptides or nucleic acids as targeting moieties, which may contain much better characteristics, through several methods including phage display ${ }^{[37]}$ and systematic evolution of ligands by exponential enrichment ${ }^{[38]}$. These have shown their efficacy in a development perspective, which may largely increase the brain targeting effect of drug delivery systems.

\section{Acknowledgments}

We are very grateful to Prof Yao-cheng RUI, Department of Pharmacology, School of Pharmacy, The Second Military Medical University, China, for providing the bEnd.3 cell line. We also acknowledge Dr Jian-hua ZHU, School of Pharmacy, Fudan University, China, for his help on radioactive element 
labeling and detecting.

This work was supported by National Basic Research Program of China (973 Program) 2007CB935800, National Natural Science Foundation of China (30762544), National Key Program of Pharmaceutical Creation and Development (2009ZX09310-006), and the ChunTsung Foundation for undergraduate of China.

\section{Author contribution}

Xin-guo JIANG designed the research; Hui-le GAO, Li FAN, and Bing-xian WU performed the research; Zhi-qing PANG and Kai-li HU contributed new reagents and analytic tools; Hui-le GAO analyzed the data; and Hui-le GAO, Zhi-qing PANG, and Kai-li HU wrote the paper.

\section{References}

1 Pardridge WM. Blood-brain barrier delivery. Drug Discov Today 2007; 12: 54-61.

2 Pardridge WM. Drug targeting to the brain. Pharm Res 2007; 24: 1733-44.

3 Begley DJ. Delivery of therapeutic agents to the central nervous system: the problems and the possibilities. Pharmacol Ther 2004; 104: $29-45$.

4 Liu X, Chen C. Strategies to optimize brain penetration in drug discovery. Curr Opin Drug Disc 2005; 8: 505-12.

5 Hu KL, Li JW, Shen YH, Lu W, Gao XL, Zhang QZ, et al. Lactoferrinconjugated PEG-PLA nanoparticles with improved brain delivery: In vitro and in vivo evaluations. J Control Release 2009; 134: 55-61.

6 Garcia-Garcia E, Andrieux K, Gilb S, Couvreur P. Colloidal carriers and blood brain barrier (BBB) translocation: away to deliver drugs to the brain? Int J Pharm 2005; 298: 274-92.

7 Boer AG, Gaillard PJ. Strategies to improve drug delivery across the blood brain barrier. Clin Pharmacokinet 2007; 46: 553-76.

8 Calvo P, Gouritin B, Chacun H, Desmaële D, D'Angelo J, Noel JP. Long circulating PEGylated polycyanoacrylate nanoparticles as new drug carrier for brain delivery. Pharm Res 2001; 18: 1157-66.

9 Nuijens JH, Berkel PHC, Schanbacher FL. Structure and biological actions of lactoferrin. J Mammary Gland Biol 1996; 3: 285-95.

10 Qian ZM, Li HY, Sun HZ, Ho K. Targeted drug delivery via the transferrin receptor-mediated endocytosis pathway. Pharmacol Rev 2002; 54: 561-87.

11 Buchanan J. A structural comparison of human serum transferrin and human lactoferrin. Biometals 2007; 20: 249-62.

12 Fillebeen C, Descamps L, Dehouck MP, Fenart L. Receptor-mediated transcytosis of lactoferrin through the blood-brain barrier. J Biol Chem 1999; 274: 7011-7.

13 Moos T, Morgan EH. Transferrin and transferrin receptor function in brain barrier systems. Cell Mol Neurobiol 2000; 20: 77-95.

14 Suzuki YA, Lopez V, Lönnerdal B. Mammalian lactoferrin receptors: structure and function. Cell Mol Life Sci 2005; 62: 2560-75.

15 Huang RQ, Ke WL, Qu YH, Zhu JH, Pei YY, Jiang C. Characterization of lactoferrin receptor in brain endothelial capillary cells and mouse brain. J Biomed Sci 2007; 14: 121-8.

16 Kawamata T, Tooyama I, Yamada T, Walker DG, McGeer PL. Lactotransferrin immunocytochemistry in Alzheimer and normal human brain. Am J Pathol 1993; 142: 1574-85.

17 Faucheux BA, Nillesse N, Damier P, Spik G, Mouatt-Prigent A, Pierce $A$, et al. Expression of lactoferrin receptors is increased in the mesencephalon of patients with Parkinson disease. Proc Natl Acad Sci USA 1995; 92: 9603-7.
18 Grau AJ, Willig V, Fogel W, Werle E. Assessment of plasma lactoferrin in Parkinson's disease. Mov Disord 2001; 16: 131-4.

19 Inoue T, Cavanaugh PG, Steck PA, Brunner N, Nicolson GL. Differences in transferrin response and numbers of transferrin receptors in rat and human mammary carcinoma lines of different metastatic potentials. J Cell Physiol 1993; 156: 212-7.

20 Ji B, Maeda J, Higuchi M. Pharmacokinetics and brain uptake of lactoferrin in rats. Life Sci 2006; 78: 851-5.

21 Discher BM, Won YY, Ege DS, Lee J, Bates FS, Discher DE, et al. Polymersomes: tough vesicles made from diblock copolymers. Science 1999; 284: 1143-6.

22 Discher DE, Eisenberg A. Polymer vesicles. Science 2002; 297: 967-73.

23 Pang ZQ, Lu W, Gao HL, Hu KL, Chen J, Zhang CL, et al. Preparation and brain delivery property of biodegradable polymersomes conjugated with OX26. J Control Release 2008; 128: 120-7.

24 Photos PJ, Bacakova L, Discher B, Bates FS, Discher DE. Polymer vesicles in vivo: correlations with PEG molecular weight. J Control Release 2003; 90: 323-34.

25 Huwyler J, Wu DF, Pardridge WM. Brain drug delivery of small molecules using immunoliposomes. Proc Natl Acad Sci USA 1996; 93: 14164-9.

26 Ellman GL. Tissue sulfhydryl groups. Arch Biochem Biophys 1959; 82: $70-7$.

27 Lu W, Zhang Y, Tan YZ, Hu KL, Jiang XG, Fu SK. Cationic albuminconjugated pegylated nanoparticles as novel drug carrier for brain delivery. J Control Release 2005; 107: 428-48.

28 Bermudez H, Brannan AK, Hammer DA, Bates FS, Discher DE. Molecular weight dependence of polymersome membrane structure, elasticity, and stability. Macromol 2002; 35: 8203-8.

29 Davda J, Labhasetwar V. Characterization of nanoparticle uptake by endothelial cells. Int J Pharm 2002; 233: 51-9.

30 Panyam J, Sahoo SK, Prabha S, Bargar T, Labhasetwar V. Fluorescence and electron microscopy probes for cellular and tissue uptake of poly $(D, L$-lactide-coglycolide) nanoparticles. Int J Pharm 2003; 262: 1-11.

31 Hunt CA, MacGregor RD, Siegel RA. Engineering targeted in vivo drug delivery: I. The physiological and physicochemical principles governing opportunities and limitations. Pharmaceut Res 1986; 3: 333-44.

32 Pepper MS, Tacchini-Cottier F, Sabapathy TK, Montesano R, Wagner EF. A model for haemangiomas and other vascular tumours. In: $R$ Bicknell CE Lewis, N Ferrara (Eds), Tumour Angiogenesis, Endothelial Cells Transformed by Polyomavirus Middle T Oncogene. Oxford, UK, Oxford University Press, 1997; p 309-31.

33 Brown RC, Morris AP, O'Neil RG. Tight junction protein expression and barrier properties of immortalized mouse brain microvessel endothelial cells. Brain Res 2007; 30: 17-30.

34 Sawyer ST, Krantz SB. Transferrin receptor number, synthesis, and endocytosis during erythropoietin-induced maturation of Friend virusinfected erythroid cells. J Biol Chem 1986; 261: 9187-95.

35 Milane A, Tortolano L, Fernandez L, Bensimon G, Meininger V, Farinotti R. Brain and plasma riluzole pharmacokinetics: effect of minocycline combination. J Pharm Pharmaceut Sci 2009; 12: 209-17.

36 Jones AR, Shusta EV. Blood-brain barrier transport of therapeutics via receptor-mediation. Pharmaceut Res 2007; 24: 1759-71.

37 Schluesener HJ, Xianglin T. Selection of recombinant phages binding to pathological endothelial and tumor cells of rat glioblastoma by in vivo display. J Neurol Sci 2004; 224: 77-82.

38 Yang Y, Yang D, Schluesener HJ, Zhang Z. Advances in SELEX and application of aptamers in the central nervous system. Biomol Eng 2007; 24: 583-92. 